\title{
Dieta hiperlipídica com farinha de soja como fonte proteica: utilização na seleção de ratos propensos e resistentes à obesidade 1
}

\author{
High-fat diet using soybean meal as protein \\ source: use for selecting rats prone \\ and resistant to obesity
}

Ludimila Canuto $\mathrm{CABEÇO}^{2}$

Mayumi AKIBA ${ }^{2}$

Marcela Silva CALSA ${ }^{2}$

Denise Rangel da Silva SARTORI ${ }^{2}$

Maria de Lourdes Mendes VICENTINI-PAULINO²

Daniela Felipe PINHEIRO2

R E S U M O

\section{Objetivo}

Desenvolver uma dieta hiperlipídica de baixo custo, tendo farinha de soja como fonte proteica, que seja eficiente na seleção de ratos propensos e resistentes à obesidade e que permita alcançar fenótipo obeso nos animais propensos. Além desses requisitos, a dieta deve ser palatável e não rejeitada a curto prazo pelo animal.

\section{Métodos}

A dieta proposta foi obtida misturando-se leite condensado $(15,5 \%)$, amendoim $(18,5 \%)$, farinha de soja $(20,0 \%)$, óleo de milho $(6,0 \%)$, ração Bio Tec $(30,0 \%)$ e bolacha wafer de chocolate $(10,0 \%)$. A mistura foi peletizada e submetida à análise bromatológica. A dieta foi ofertada a ratos Wistar durante uma semana; posteriormente, os animais foram divididos em três grupos, de acordo com o ganho de peso. O terço superior foi considerado propenso à obesidade e o terço inferior, resistente à obesidade. Após 80 dias de oferta da dieta, os animais foram sacrificados e foram quantificados o peso corpóreo, consumo alimentar, gorduras retroperitoneal, periepididimal, de carcaça e gorduras totais.

\section{Resultados}

Verificou-se que a dieta apresentava 5,31 kcal/g, com a seguinte composição: 22,3\% de gordura, 22,2\% de proteína, $15,9 \%$ de fibra, estimando-se $35,7 \%$ de carboidrato. Ratos propensos à obesidade, alimentados

\footnotetext{
1 Artigo elaborado a partir da dissertação de L.C. CABEÇO, intitulada "Flexibilidade fenotípica do trato gastrointestinal de ratos propensos e resistentes à obesidade". Universidade Estadual Paulista Júlio de Mesquita Filho; 2008.

2 Universidade Estadual Paulista Júlio de Mesquita Filho, Instituto de Biociências de Botucatu, Departamento de Fisiologia. Campus de Botucatu, Distrito de Rubião Júnior, s/n., 18618-000, Botucatu, SP, Brasil. Correspondência para/Correspondence to: M.L.M. VICENTINI-PAULINO. E-mail: <mlvpauli@ibb.unesp.br>.
} 
418 | L.C. CABEÇO et al.

por 87 dias com a dieta hipercalórica, apresentaram peso corpóreo, gorduras retroperitoneal, periepididimal e totais significativamente maiores do que animais resistentes à obesidade $(p<0,05)$. O consumo de alimentos também foi maior em animais propensos $(p<0,05)$.

Verificou-se também que a substituição da caseína pela farinha de soja, como componente proteico da ração, levou à diminuição de $96,0 \%$ no custo do estudo.

\section{Conclusão}

A dieta formulada com farinha de soja apresentou custo reduzido e foi capaz de desenvolver o fenótipo obeso em ratos propensos, à semelhança do observado na literatura com outras dietas.

Termos de indexação: Depósito de gorduras. Dieta hiperlipídica. Farinha de soja. Propensão e resistência à obesidade. Ratos.

\section{A B S T R A C T}

\section{Objective}

The objective was to develop a high-fat, low cost diet, using soybean meal as protein source. This diet should effectively discriminate between rats prone and resistant to obesity and allow the obese phenotype to be achieved in the animals that are prone to obesity. Furthermore, the diet must be palatable and not be rejected by the animal in the short run.

\section{Methods}

The chow was obtained by mixing the following ingredients: condensed milk (15.5\%), peanuts (18.5\%), soybean meal (20.0\%), corn oil (6.0\%), Bio Tec chow (30.0\%) and chocolate wafer cookies (10.0\%). In order to make it appropriate for rats, the mixture was pelleted and subjected to food analysis. The chow was offered to Wistar rats for a week. The animals were subsequently separated according to weight gained. The upper third group was considered prone to obesity and the lower third group was considered resistant to obesity. The animals were sacrificed 80 days later to determine body weight, food intake, retroperitoneal, periepididymal and carcass fats and total fats.

\section{Results}

Food analysis found that the chow had an energy density of $5.31 \mathrm{Kcal} / \mathrm{g}, 22.3 \%$ fat, 22.2\% protein, $15.9 \%$ fiber and $35.7 \%$ carbohydrates. After being fed for 87 days with the high-fat diet, obesity-prone rats had higher body weight and retroperitoneal, periepididymal and total fats than obesity-resistant animals $(p<0.05)$. Food intake was also higher among obesity-prone rats $(p<0.05)$. The replacement of casein by soybean meal as protein source reduced the cost of the study by $96.0 \%$.

\section{Conclusion}

The substitution of casein by soybean meal in a high-fat diet allows cost reduction and the identification of obese-prone rats. Continuous use of this high-fat diet resulted in the development of the obese phenotype, as seen with other diets used in the literature.

Indexing terms: Fat deposit. Hyperlipidic diet. Soya flour. Obesity-prone and obesity-resistant. Rats.

\section{N T R O D U ÇÃ O}

A obesidade é atualmente considerada epidemia mundial e suas causas são atribuídas a hábitos modernos - como consumo de alimentos altamente calóricos e sedentarismo - associados à predisposição genética do indivíduo. Muitas enfermidades, como hipertensão, diabetes, doenças cardiovasculares, apneia, neoplasias, doenças endócrinas e problemas pulmonares estão relacionadas ao sobrepeso ${ }^{1,2}$.
Por esse motivo, a obesidade se tornou alvo de muitos trabalhos científicos e hoje é extensamente estudada; muitos dos estudos experimentais exigem o emprego de dietas altamente energéticas $^{3-8}$.

De fato, muitas dietas são manipuladas na tentativa de se alcançar os índices de gordura e açúcar ideais para indução da obesidade em animais de laboratório. Entre elas, a dieta hiperlipídica, cuja administração por tempo prolongado 
resulta em balanço energético positivo e obesidade em animais de experimentação. Sendo assim, essa dieta foi considerada um modelo adequado para simulação ou estudo da obesidade humana ${ }^{7}$.

A proposta de uma dieta experimental deve levar em consideração não só a quantidade de calorias, mas também suas características físicas e de palatabilidade. De fato, existem evidências de que as propriedades físicas da dieta podem influenciar o consumo de alimentos. Embora alimentos pastosos, com alto teor de gordura e carboidratos, e alimentos hidratados sejam bem aceitos pelos ratos ${ }^{9,10}$, deve-se levar em conta o hábito alimentar dos animais, principalmente em experimentos de longo prazo. É importante, portanto, que ratos, por serem roedores, sejam alimentados com pellets para que mantenham seu hábito alimentar e não passem a apresentar comportamentos indesejáveis, como o de roer partes da gaiola de manutenção.

A alta palatabilidade da dieta hiperlipídica é considerada um dos fatores desencadeadores da hiperfagia dos animais tratados com esse tipo de ração ${ }^{11}$. No entanto, existem diferenças na preferência alimentar entre ratos propensos e resistentes à obesidade. De fato, ratos Osborne-Mendel, propensos a desenvolver a obesidade, têm maior palatibilidade por alimentos ricos em gordura, enquanto os ratos S5B/PL, resistentes a desenvolver a obesidade com dieta rica em gordura, preferem alimentos com alto conteúdo de carboidrato' ${ }^{12}$. Também dentro de uma população de animais aparentemente homogênea, cujos indivíduos não tenham sofrido manipulação genética, é possível observar consumo diferenciado ${ }^{4}$.

Em experimentos realizados com ratos, foi observado que mesmo o emprego de uma dieta altamente palatável e energética não garante que todos animais se tornem obesos, pois muitos deles ganham peso como os animais controles, que são alimentados com dieta padrão ${ }^{13-15}$. A possível explicação está na evidência de que, também como ocorre entre homens, existem animais propensos e animais resistentes à obesidade ${ }^{13-15}$. Esses achados têm levado os pesquisadores da obesidade a adotarem o modelo experimental que permite identificar animais propensos e resistentes à obesidade.

MacLean et al. ${ }^{16}$ propuseram identificar ratos propensos e obesos analisando o ganho de peso após uma semana de tratamento com dieta de alto conteúdo de gordura. Depois desse período de tratamento, os animais são separados em três subgrupos de acordo com o ganho de peso obtido. O terço superior é considerado propenso à obesidade e o terço inferior, resistente à obesidade. Com a continuidade do tratamento, os animais propensos ganham mais peso e acumulam mais tecido adiposo do que os animais resistentes à obesidade, com a continuidade do tratamento.

A associação entre o consumo de dieta hipercalórica ou hiperlipídica e a predisposição genética, portanto, tem sido considerada, hoje em dia, o modelo mais realista e apropriado para estudos da obesidade ${ }^{17-19}$.

As dietas experimentais mais frequentemente utilizadas para provocar obesidade são formuladas com o acréscimo de itens lipídicos e energéticos à ração padrão. Entre esses itens estão amendoim, leite condensado, biscoitos tipo waffer ou "maizena", óleo e chocolate7,8.

A maior parte dessas dietas usa como fonte proteica ${ }^{3,5,12,13}$ a caseína, um componente que eleva o custo da ração, principalmente se comparada à farinha de soja, cujo valor é aproximadamente 20 vezes menor. É interessante, portanto, o estudo de fontes proteicas alternativas para a composição das dietas utilizadas em pesquisas.

Com o objetivo de baratear o custo, na composição da ração animal são utilizados a soja e seus subprodutos ${ }^{20,21}$. A soja tem sido utilizada também na alimentação humana, até mesmo em dietas enterais ${ }^{21}$. O uso de tal ingrediente se justifica principalmente pelo seu alto valor nutritivo devido ao conteúdo de proteínas adequadas às necessidades nutricionais de adultos.

Com relação à forma de oferecimento, verificou-se que a farinha de soja apresenta maior conteúdo proteico do que os grãos, embora em 
ambos a digestibilidade de proteína por ratos seja de $88 \%{ }^{21}$.

Tendo em vista o interesse na utilização de ração de preço mais acessível e a dificuldade de se obter $100 \%$ de ratos obesos no final do tratamento com dietas hiperenergéticas ${ }^{17-20}$, nosso objetivo foi desenvolver uma dieta hiperlipídica de baixo custo, tendo farinha de soja como fonte proteica, que seja eficiente na seleção de ratos propensos e resistentes à obesidade e que permita alcançar fenótipo obeso nos animais propensos. Além desses requisitos, a dieta deve ser palatável e não rejeitada a curto prazo pelo animal.

\section{M É TO D O S}

A dieta foi preparada no dietário do Laboratório Experimental do Departamento de Clínica Médica da Universidade Estadual Paulista Júlio de Mesquita Filho (UNESP), em Botucatu. A dieta proposta foi baseada na de Nascimento et al..$^{19} \mathrm{com}$ a introdução de farinha de soja como fonte proteica - e foi constituída por leite condensado, amendoim - torrado em forno e posteriormente triturado -, farinha de soja, óleo de milho, ração para roedores Bio $\operatorname{Tec}^{\circledR}$ e bolacha de chocolate tipo waffer (Tabela 1). A ração Bio Tec ${ }^{\circledR}$ e a bolacha waffer também foram trituradas previamente.

Todos os ingredientes foram misturados em misturadeira elétrica. Para cada $12 \mathrm{~kg}$ de dieta manipulada, foram adicionados $700 \mathrm{~mL}$ de água. Após a mistura, a dieta pastosa foi colocada numa máquina peletizadora. Os pellets formados foram então colocados na estufa por período de 24 horas para secagem.

Tabela 1. Constituição da dieta hiperlipídica. Botucatu (SP), 2007.

\begin{tabular}{lc}
\hline Ingredientes & Proporção (\%) \\
\hline Amendoim & 18,5 \\
Bolacha waffer & 10,0 \\
Leite condensado & 15,5 \\
Farinha de soja & 20,0 \\
Óleo de milho & 6,0 \\
Ração Bio-Tec & 30,0 \\
\hline
\end{tabular}

A ração peletizada foi submetida à análise bromatológica, segundo método de Weende ${ }^{22}$, no Laboratório de Bromatologia da Faculdade de Medicina Veterinária e Zootecnia da UNESP-Botucatu. A quantidade de energia foi determinada por bomba calorimétrica no laboratório de Química do Departamento de Bioquímica do Instituto de Biociências UNESP-Botucatu. A dieta foi ofertada a ratos Wistar inicialmente na seleção de animais propensos e resistentes à obesidade e, posteriormente, ao longo do tratamento.

Para a seleção, 48 ratos Wistar machos, com 54 dias de idade, pesando em Média $M=258$,4, Desvio-Padrão - $D P=28$ g, foram alimentados ad libitum com dieta hiperlipídica por um período de sete dias; posteriormente foi avaliado o ganho de peso dos animais. Os 16 animais que ganharam mais peso (terço superior) foram classificados como propensos à obesidade, enquanto os 16 que ganharam menos peso (terço inferior) foram classificados como resistentes. Após a seleção, os animais de ambos os grupos foram alimentados ad libitum por 80 dias com a dieta hiperlipídica. Os animais do grupo intermediário não foram utilizados no experimento, sendo disponibilizados para aulas práticas.

Durante todo o experimento, os animais obesos e resistentes foram mantidos em gaiolas individuais, em ambiente com controle de temperatura $\mathrm{M}=24^{\circ} \mathrm{C}, \mathrm{DP}=2^{\circ} \mathrm{C}$ e fotoperíodo ( $12 \mathrm{~h}: 12 \mathrm{~h}$ ), com livre acesso à água e à dieta hiperlipídica. $\mathrm{O}$ peso corpóreo foi quantificado semanalmente, e o consumo, de três em três dias, durante as dez semanas de fornecimento da dieta.

Para a quantificação do tecido adiposo, após sacrifício dos animais, por decapitação, o abdômen foi aberto, as vísceras e as gorduras periepididimal e retroperitoneal foram retiradas e pesadas. Em seguida, o conteúdo de gordura de carcaça foi determinado gravimetricamente após extração pelo método de Jansen et al..23.

Os grupos foram comparados por teste $t$ para duas amostras independentes. O nível de significância adotado foi de 5\%. 
Este trabalho foi aprovado pela comissão do Comitê de Ética Local em experimentação animal (Instituto de Biociências, UNESP-Botucatu - protocolo n 024/06-CEEA).

\section{RES U LTA D O S}

A análise bromatológica e o conteúdo energético da dieta estão demonstrados na Tabela 2.

Ambos os grupos apresentaram pequena variabilidade na ingestão diária da dieta ao longo do tratamento (Figura 1). Animais propensos à obesidade, no entanto, apresentaram ingestão total de $M=1385,9, D P=122 \mathrm{~g}$, um valor estatisticamente diferente $(p=0,00003)$ da média observada em animais resistentes: $M=1192,9, D P=99 g$.

Após uma semana de tratamento, o ganho de peso foi diferente entre os grupos Propensos $(\mathrm{P})$ e Resistentes $(\mathrm{R})$ à obesidade. $\mathrm{O}$ ganho de peso do grupo $P$ foi de $M=61,4, D P=7,6$ g, estatisticamente maior $(p=0,0000002)$ do que dos animais resistentes, que foi de $M=42,6, D P=8,2 \mathrm{~g}$. A porcentagem de ganho de peso do Grupo $P$ foi de $M=22,6 \%, D P=3,5$, enquanto do grupo $R$ foi de $M=16,1 \%, D P=4,7$.

A diferença no ganho de peso entre grupos foi mantida ao longo das 10 semanas de tratamento (Figura 2), sendo sempre maior para o grupo P. O ganho de peso total, após 87 dias de consumo de dieta hiperlipídica, foi de $M=256,2, D P=40 \mathrm{~g}$ para os animais propensos e de $\mathrm{M}=187,8, \mathrm{DP}=35 \mathrm{~g}$

Tabela 2. Composição bromatológica e conteúdo energético da ração. Botucatu (SP), 2007.

\begin{tabular}{lc}
\hline Componentes & Composição (\%) \\
\hline Gordura & 22,3 \\
Proteína & 22,2 \\
Carboidrato* & 35,7 \\
Fibra & 15,9 \\
Cinzas & 3,9 \\
Energia & $5,31 \mathrm{kcal} / \mathrm{g}$ \\
\hline
\end{tabular}

*Estimativa. para os animais resistentes $(p=0,0003)$. Ao final do tratamento, o grupo propenso apresentou peso corpóreo médio de $M=530,6, D P=58 \mathrm{~g}$, que foi estatisticamente maior $(p=0,00009)$ que 0 apresentado pelo grupo resistente: $M=436,7$, $\mathrm{DP}=33 \mathrm{~g}$.

O grupo propenso apresentou maior valor de gordura retroperitoneal $(p=0,007)$ e periepididimal $(p=0,005)$ do que os animais resistentes. A soma das gorduras retroperitoneal, periepidi-

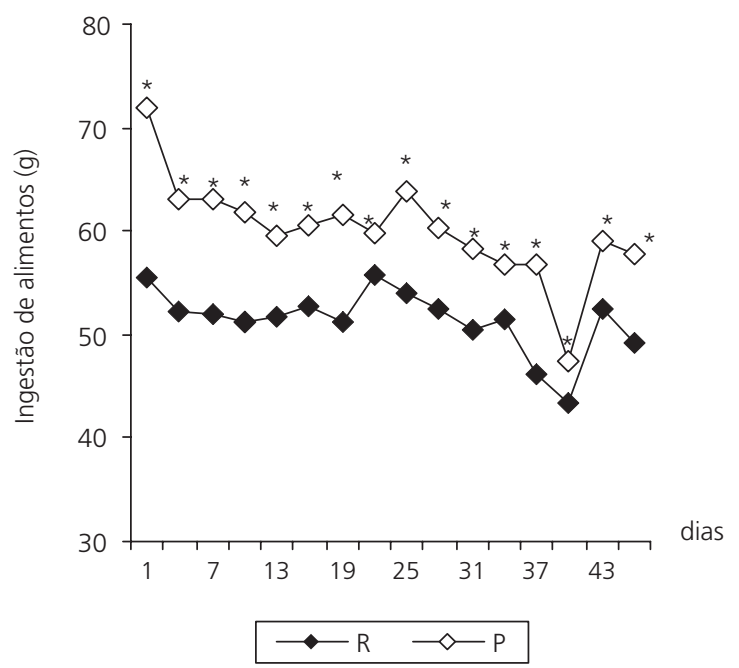

Figura 1. Média e desvio-padrão da ingestão alimentar ( $\mathrm{g}$ ) de animais propensos $(P)$ e resistentes $(R)$ à obesidade. Consumo acumulado de três dias. Botucatu (SP), 2007.

Nota: Asteriscos indicam diferença significativa entre grupos $(p<0,05)$.

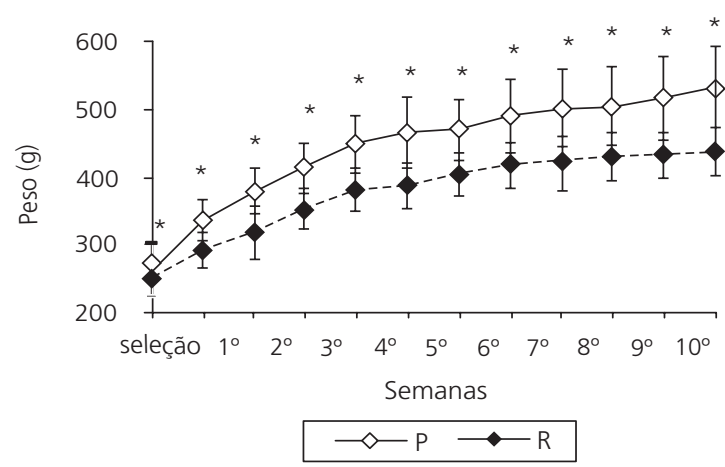

Figura 2. Média, desvio-padrão do peso corpóreo (g) de animais propensos $(P)$ e resistentes $(R)$ à obesidade. Botucatu (SP), 2007.

Nota: Asteriscos indicam diferença significativa entre grupos $(p<0,05)$. 
dimal e de carcaça foi também maior $(p=0,00002)$ no grupo propenso (Figura 3).

A utilização de farinha de soja reduziu o custo da fonte proteica, e consequentemente o custo da ração, em aproximadamente $96 \%$.

\section{I S C U S S Ã O}

A dieta hiperlipídica manipulada com farinha de soja como fonte proteica permitiu a identificação de animais propensos e resistentes à obesidade, à semelhança do relatado na literatura quando do emprego de dietas com outras composições ${ }^{6,14-17,24}$. De fato, após uma semana de tratamento, já pudemos separar animais que ao fim do experimento teriam maior peso corpóreo.

Também o ganho de peso médio com a ração aqui empregada, que foi de 20,5g/semana, está bastante próximo ao apresentado com o uso de dietas hiperlipídicas com caseína como fonte proteica, cujos valores variam de 18,8 a $20 \mathrm{~g} / \mathrm{se}-$ mana $a^{6,25}$.

O ganho de peso observado pode ser atribuído a um maior depósito de gordura, uma vez que os animais propensos apresentaram maior quantidade de gorduras totais, gordura periepididimal e retroperitoneal do que os animais resistentes. O maior peso corpóreo e de gordura apresentado pelo grupo propenso são sinais indicadores de que a dieta permitiu o desenvolvimento do fenótipo obeso. O aumento desses mesmos tecidos foi observado em trabalhos e são considerados indicativos de obesidade ${ }^{26-28}$.

Talvez a farinha de soja tenha contribuído para esse maior depósito de gordura, uma vez que foi verificado que ratos alimentados com dietas isoenergéticas contendo proteína de soja apresentam maior tolerância à glicose e maior sensibilidade à insulina do que animais cuja fonte proteica na dieta é a caseína ${ }^{29}$.

A pequena variabilidade na ingestão, dentro de cada grupo, na maior parte do tratamento, indica que a dieta foi bem aceita e que é palatável aos animais. Além disso, não houve diminuição da ingestão, como ocorre com outras dietas hiperlipídicas que levam mais rapidamente à saciedade e induzem a um tempo maior de saciação 29,30 .

A introdução de farinha de soja na formulação da ração pôde ser feita sem que houvesse alteração significativa da proporção dos demais

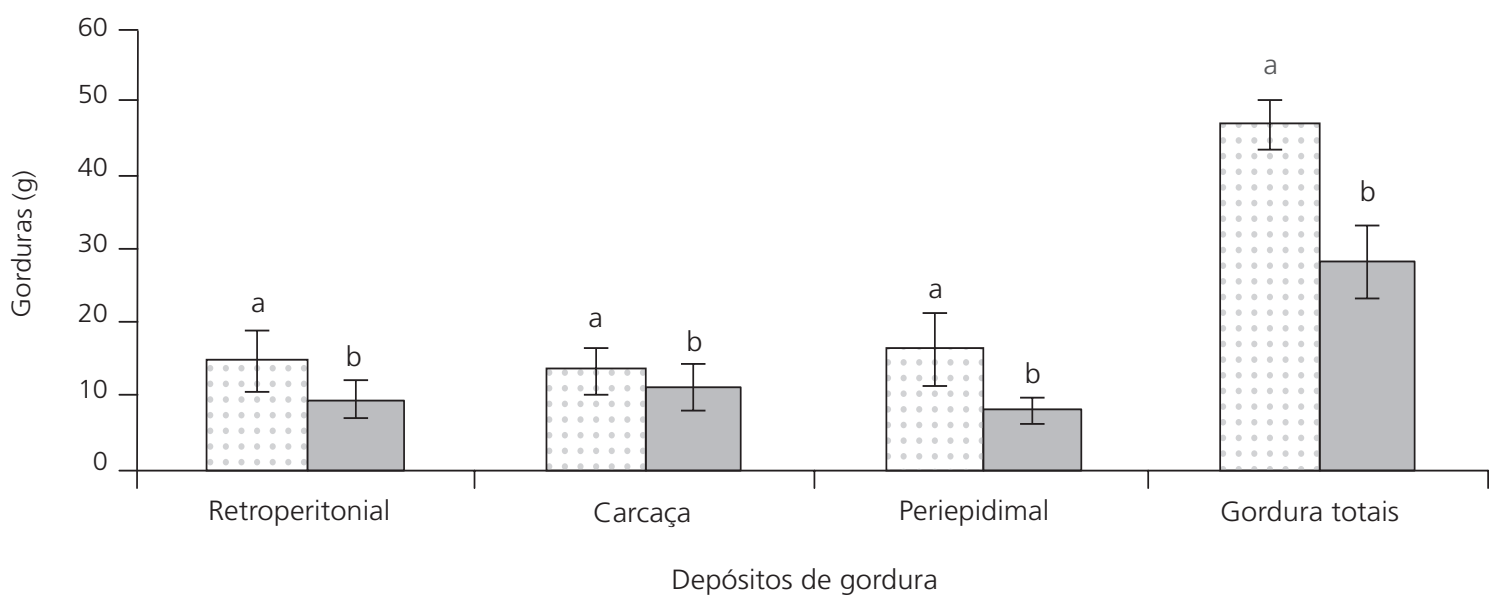

Figura 3. Peso das gorduras retroperitoneal, de carcaça, periepididimal e soma das gorduras. Botucatu, SP, 2007. Nota: Letras minúsculas indicam diferença significativa $(p<0,05)$ entre grupos propensos $(P)$ e resistentes $(R)$ à obesidade. 
componentes, tendo sido obtidos níveis de 20,0\% de gordura e $20,0 \%$ de proteína, valores semeIhantes ao das dietas utilizadas por outros pesquisadores, que variam entre $20,0 \%$ e $32,7 \%$ de gordura e $20,0 \%$ e $29,3 \%$ de proteína $a^{6,10}$.

A diferença de preço entre caseína e farinha de soja muda acentuadamente o custo da ração quando se usa uma ou outra fonte proteica. De fato, considerando que os demais ingredientes foram utilizados em proporções bastante aproximadas às dietas hiperlipídicas com caseína, podemos concluir que a diferença entre o preço da caseína e da farinha de soja tem reflexo direto no preço das duas dietas.

Tomados em conjunto, esses resultados mostram a viabilidade do emprego dessa dieta em estudos de obesidade, com a vantagem de ser mais acessível por seu baixo custo.

\section{O N C L U S Ã O}

Conclui-se que a dieta proposta pode ser utilizada como alternativa às dietas hiperenergéticas encontradas na literatura, uma vez que promove o ganho de peso e de tecido gorduroso de animais propensos, além de não diminuir a ingestão de alimentos. Além do mais, o emprego de farinha de soja como fonte proteica, no lugar de caseína, permite significativa redução do custo da dieta.

\section{A GRADECIMENTOS}

Aos técnicos do biotério do Departamento de Fisiologia do Instituto de Biociências da Universidade Estadual Paulista Júlio de Mesquita Filho, do dietário do Depto Experimental das Clínicas Médicas e do Laboratório de Bromatologia do Depto de Zootecnia da Universidade Estadual Paulista Júlio de Mesquita Filho.

\section{COLABORADORES}

L.C. CABEÇO contribuiu com a elaboração do projeto de pesquisa, da estratégia experimental, da elaboração e da manipulação da dieta da manutenção dos animais no biotério da coleta de dados da tabulação e da discussão dos resultados e da elaboração do artigo. M. AKIBA e M.S CALSA participaram da manipulação da dieta, da manutenção dos animais no biotério e da coleta de dados. D.F. PINHEIRO participou da estratégia experimental e da orientação das técnicas laboratoriais. D.R. SARTORI participou da coleta de dados e da orientação das técnicas laboratoriais. M.L.M. VICENTINI-PAULINO participou da elaboração do projeto de pesquisa, da tabulação e da discussão dos resultados e da elaboração do artigo.

\section{REFERÊ NCIAS}

1. Pereira LO, Francischi RP, Lancha AH. Obesidade: hábitos nutricionais, sedentarismo e resistência à insulina. Arq Bras Endocrinol Metab. 2003; 47 (2):111-27.

2. Korner J, Aronne $L$ J. The emerging science of body weight regulation and its impact on obesity treatment. J Clin Invest. 2003; 111(5):565-70.

3. Harris RBS. Factors influencing energy intake of rats fed either a high-fat or a fat mimetic diet. Int J Obes. 1994; 18(9):632-40.

4. Chang S, Graham B, Yakubu F, Lin D, Peters JC, Hill JO. Metabolic differences between obesityprone and obesity-resistant rats. Am J Physiol Reg Int Comp Physiol. 1990; 259(6):1103-10.

5. Woods SC, Seeley RJ, Rushing PA, Dálessio D, Tso P. A controlled high-fat diet induces an obese syndrome in rats. J Nutr. 2003; 133(5):1081-7.

6. Estadella D, Oyama LM, Dâmaso AR, Ribeiro EB, Oller CM. Effect of palatable hyperlipidic diet on lipid metabolism of sedentary and exercised rats. Nutrition. 2004; 20(2):218-24.

7. Duarte ACGO, Fonseca DF, Soave CF, Fiorese MS, Dâmaso AR. Dieta hiperlipídica e capacidade secretória de insulina em ratos. Rev Nutr. 2006; 19(3): 341-8. doi: 10.1590/S1415-52732006000300005.

8. Lucas F, Ackroff K, Sclafani A. Dietary fat-induced hyperphagia in rats as a function of fat type and physical form. Physiol-Behav. 1989; 45(5):937-46.

9. Ackroff K, Lucas F, Sclafani A. Flavor preference conditioning as a function of fat source. Physiol-Behav. 2005; 85(4):448-60.

10. Dourmashkin JT, Chang GQ, Hill JO, Gayles EC, Fried SK, Leibowitz SF. Model for predicting and phenotyping at normal weight the long-term propensity for obesity in Sprague-Dawley rats. Physiol Behav. 2006; 87(4):666-78.

11. Greenberg D, McCaffery J, Potack JZ, Bray GA, York DA. Differential satiating effects of fats in the small 
424 | L.C. CABEÇO et al.

intestine of obesity-resistant and obesity-prone rats. Physiol Behav.1999; 66(4):621-26.

12. Levin BE, Arcuate NPY. Neurons and energy homeostasis in diet-induced obese and resistant rats. Am J Physiol Regul Integr Comp Physiol. 1999; 276(2):R382-R7.

13. Levin BE, Hogan S, Sullivan AC. Initiation and perpetuation of obesity and obesity resistance in rats. Am J Physiol Regul Integr Comp Physiol. 1989; 256(3):R766-R71

14. Levin BE, Keesey RE. Defense of differing body weight set points in diet-induced obese and resistant rats. Am J Physiol Regul Integr Comp Physiol. 1998; 274(3):R412-9.

15. York DA. Genetic models of animal obesity. In: Björntorp P, Brodoff BN, editors, Obesity. Philadelphia: J.B. Lippincott Company; 1992. p.233-40.

16. MacLean PS, Higgins JA, Johnson GC, FlemingElder BK, Peters JC, Hill JO. Metabolic adjustments with the development, treatment, and recurrence of obesity in obesity-prone rats. Am J Physiol Regul Integr Comp Physiol. 2004; 287(2):R288-97.

17. Marques-Lopes I, Marti A, Moreno-Aliaga A. Aspectos genéticos da obesidade. Rev Nutr. 2004; 17(3):327-38. doi: 10.1590/S1415-5273200400 0300006

18. Willet $\mathrm{W}$. Is dietary fat a major determinant of body fat? Am J Clin Nutr. 1998; 67:556-62.

19. Nascimento AF, Sugisaki MM, Leopoldo A, LimaLeopoldo AP, Luvizotto RAM, Nogueira CR. A Hypercaloric pellet-dietcycles induces obesity and co-morbidities in Wistar Rats. Arq Bras Endocrinol Metabol. 2008; 52(8):968-74. doi: 10.1590/5000 4-27302008000600007.

20. Monteiro JBR, Costa NMB, Milagres EKH. Avaliação da qualidade proteica de dois formulados em pó, à base de soja enriquecidos com zinco, selênio e magnésio para utilização em nutrição enteral. Ciênc Tecnol Aliment. 2004; 24(1):6-10. doi: 10.1590/S0101-20612004000100002.

21. Silva MS, Naves MMV, Oliveira RBO, Leite SM. Composição química e valor proteico do resíduo de soja em relação ao grão de soja. Cienc Tecnol Aliment. 2006; 26(3):571-6. doi: 10.1590/S0101-20 612006000300014.

22. Association of Official Analytical Chemists. Official Methods of Analysis. 15 $5^{\text {th }}$ ed. Arlington: AOAC; 1990.

23. Jansen GR, Zanetti ME, Hutchison CF. Studies in lipogenesis in vivo. Fatty acid and cholesterol synthesis during starvation and refeeding. Biochem J. 1966; 101(3):811-8.

24. Levin BE, Dunn-Meynell ADM. Defense of body weight against chronic caloric restriction in obesityprone and -resistant rats. Am J Physiol Regul Integr Comp Physiol. 2000; 278(1):R231-7.

25. Commerford SR, Pagliassotti MJ, Melby CL, Wei Y, Hill JO. Inherent capacity for lipogenesis or dietary fat retention is not increased in obesity-prone rats. Am J Physiol Regul Integr Comp Physiol. 2001; 280(6):1680-7.

26. Levin BE, Dunn-Meynell AA, Ricci MR, Cummings $\mathrm{DE}$. Abnormalities of leptin and ghrelin regulation in obesity-prone juvenile rats. Am J Physiol End Metab. 2003; 285(5):949-57.

27. Takes NC, Levin BE. Obesity-prone rats have preexisting defects in their counterregulatory response to insulin-induced hipoglycemia. Am J Physiol Regul Integr Comp Physiol. 2004; 287(5): 1110-5.

28. Lavigne C, Marette A, Jacques H. Cod and soy proteins compared with casein improve glucose tolerance and insulin sensitivity in rats. Am J Physiol Endocrinol Metab. 2000; 278(3):E491-E500.

29. Himaya A, Fantino M, Antoine JM, Brondel L, Louis-Sylvestre J. Satiety power of dietary fat: a new appraisal. Am J Clin Nutr. 1997; 65(5):1410-8.

30. Levin BE, Dunn-Meynell ADM. Defense of body weight depends on dietary composition and palatability in rats with diet-induced obesity. Am J Physiol Regul Integr Comp Physiol. 2002; 282(1): R46-R54.

Recebido em: 6/5/2008

Versão final reapresentada em: 30/7/2009 Aprovado em: 22/2/2010 